\title{
STEREO observations of quasi-periodically driven high velocity outflows in polar plumes ${ }^{\star}$
}

\author{
S. W. McIntosh ${ }^{1}$, D. E. Innes ${ }^{2}$, B. De Pontieu ${ }^{3}$, and R. J. Leamon ${ }^{4,5}$ \\ 1 High Altitude Observatory, National Center for Atmospheric Research, PO Box 3000, Boulder, CO 80307, USA \\ e-mail: mscott@ucar.edu \\ 2 Max-Planck Institut für Sonnensystemforschung, 37191 Katlenburg-Lindau, Germany \\ 3 Lockheed Martin Solar and Astrophysics Lab, 3251 Hanover St., Org. ADBS, Bldg. 252, Palo Alto, CA 94304, USA \\ 4 ADNET Systems Inc., NASA/GSFC, Greenbelt, MD 20771, USA \\ 5 Now at Department of Physics, Montana State University, Bozeman, MT 59717, USA
}

Received 19 November 2009 / Accepted 19 January 2010

\section{ABSTRACT}

\begin{abstract}
Context. Plumes are one of the most ubiquitous features seen at the limb in polar coronal holes and are considered to be a source of high density plasma streams to the fast solar wind.

Aims. We analyze STEREO observations of plumes and aim to reinterpret and place observations with previous generations of EUV imagers within a new context that was recently developed from Hinode observations.

Methods. We exploit the higher signal-to-noise, spatial and temporal resolution of the EUVI telescopes over that of SOHO/EIT to study the temporal variation of polar plumes in high detail. We employ recently developed insight from imaging (and spectral) diagnostics of active region, plage, and quiet Sun plasmas to identify the presence of apparent motions as high-speed upflows in magnetic regions as opposed to previous interpretations of propagating waves.

Results. In almost all polar plumes observed at the limb in these STEREO sequences, in all coronal passbands, we observe high speed jets of plasma traveling along the structures with a mean velocity of $135 \mathrm{~km} \mathrm{~s}^{-1}$ at a range of temperatures from $0.5-1.5 \mathrm{MK}$. The jets have an apparent brightness enhancement of $\sim 5 \%$ above that of the plumes they travel on and repeat quasi-periodically, with repeattimes ranging from five to twenty-five minutes. We also notice a very weak, fine scale, rapidly evolving, but ubiquitous companion of the plumes that covers the entire coronal hole limb.

Conclusions. The observed jets are remarkably similar in intensity enhancement, periodicity and velocity to those observed in other magnetic regions of the solar atmosphere. They are multi-thermal in nature. We infer that the jets observed on the plumes are a source of heated mass to the fast solar wind. Further, based on the previous results that motivated this study, we suggest that these jets originated in the upper chromosphere.
\end{abstract}

Key words. Sun: corona - solar wind - Sun: transition region

\section{Introduction}

Plumes are one of the most ubiquitous features seen at the limb in polar coronal holes (e.g., Newkirk \& Harvey 1968). In recent years they have been studied in great detail (see e.g., DeForest et al. 1997; Ofman et al. 1999; Banerjee et al. 2009, as a representative few) and are thought of as sources of dense plasma in the fast solar wind (e.g., Gabriel et al. 2003) that result from the relentless magnetoconvective forcing of the upper solar atmospheric plasma (e.g., Wang 1998; McIntosh et al. 2007).

We use high cadence observations by the STEREO spacecraft to explore the weak, fine, temporal variations of the emitting material that appear to be ubiquitous in polar plumes. We use a simple space-time data analysis technique similar to that used in the investigation of quasi-periodic intensity perturbations in active regions. McIntosh \& De Pontieu (2009a) followed from De Pontieu et al. (2009) to demonstrate that these weak, quasi-periodic perturbations in coronal imaging data are directly related with the weak, high speed Doppler velocity signals in the blue wing of several coronal emission lines. Further, these

$\star$ Five movies are only available in electronic form at http://www . aanda.org blue-wing asymmetries are co-spatial and co-temporal with high speed chromospheric jets, or "type-II" spicules (De Pontieu et al. 2007a), rooted in the magnetic footpoints of the active region. McIntosh \& De Pontieu (2009b) (and McIntosh, et al. 2010) showed that these upflows are ubiquitous in magnetic network regions (of the quiet Sun and coronal holes), again occurring quasi-periodically with repeat times ranging from a few, to several tens of minutes, and with speeds determined from the analysis of line profile asymmetries of many spectral lines (formed over a broad range of formation temperatures) that are similar to those observed in the apparent motions derived from coronal imaging data (e.g., Schrijver et al. 1999).

In the following sections we discuss the STEREO observations used and the analysis techniques employed. Based on an argument of similarity with spectroscopic and imaging features observed ubiquitously in quiet, coronal hole, and active solar plasmas alike we suggest that the quasi-periodic jets in polar plumes observed at the limb by STEREO are likely to be similarly excited, with roots in dynamic upper chromospheric activity. We conclude by discussing the implications of this new interpretation, looking forward to observational tests of the paradigm 
and closing with speculation on the impact of our interpretation on the process of solar wind acceleration.

\section{Observations and reduction}

The observations discussed in this letter were made by the EUVI/SECCHI telescopes on the STEREO spacecraft from 14:00-18:00 UT on 12-15 Feb. 2009. During the four hours of observation on each day, STEREO-A took $171 \AA$ passband images every $75 \mathrm{~s}$ that were synchronized with $304 \AA$ images of STEREO-B. The EUVI telescopes also took a slightly lower cadence (95s) synchronized sequence of STEREO-A $304 \AA$ and STEREO-B $171 \AA$ over the same period with context images in the $195 \AA$ and $284 \AA$ passbands every $10 \mathrm{~min}$. At this time STEREO-B was $47.5^{\circ}$ behind the earth and STEREO-A was $43.4^{\circ}$ ahead, yielding a total spacecraft separation angle of $91^{\circ}$.

The EUVI/SECCHI data have been calibrated with the standard SolarSoft IDL routines. They were corrected for the telescope roll angle and deprojected so that the solar B angle is zero for all images. The disk center images were then corrected for differential rotation and aligned to the start time of each sequence. We then expanded the Sun in the B images to match the radius of the A images. After using the numbers from the headers, we still found small corrections were necessary to achieve perfect co-alignment of features. The STEREO-A images required an additional rotation of $2^{\circ}$ and the Sun center in the STEREO-B images was shifted north by $5^{\prime \prime}$.

\section{Analysis and results}

We have isolated the North polar coronal hole $(\mathrm{PCH})$ region in the EUV imaging sequences shown in Fig. 1. The online edition of the journal contains movies of the 304, 171, 195, and $284 \AA$ passbands of the A spacecraft in this region. From those movies the temporal variability of the polar plume above the north polar limb $\left(x=-22^{\prime \prime}\right)$ is easily observed in all three coronal passbands. Connection of the plume to the low transition region plasma ( $304 \AA$ ) is complicated by the prominent fore- and background spicules (and macrospicules). We interpret the very weak emission seen in the $284 \AA$ passband as that of the Si VII $275 \AA$ emission line that is formed, in equilibrium, at $0.6 \mathrm{MK}$ (Mazzotta et al. 1998) and dominates the passband in quieter network regions near solar minimum.

To quantify the variability in the plume we produced a timeseries of background subtracted images by removing a 5 pixel (7.75") boxcar smoothed version of each image in the $171 \AA$ sequence from itself. As a result of this processing only the finer spatial scale fluctuations remain (cf. McIntosh \& De Pontieu 2009a). Placing a 100" long "slit" at $-22^{\prime \prime}, 940$ " normal to the limb for the $171 \AA$ STEREO-A sequence we obtained the timeseries shown in Fig. 2.

In Fig. 3 we assess the temporal variation of the emission along the slit. In the top panel we show the percentage change in the background-subtracted timeseries imaged along the slit and see the series of inclined alternating black and white stripes. The white stripes represent intensity enhancements of $\sim 5-7 \%$. The inclination of the stripes indicates the propagation speed of the disturbance along the chosen trajectory where, for reference, we show a red line on the jet starting $95 \mathrm{~min}$ into the timeseries with a velocity of $142\left( \pm 10 \mathrm{~km} \mathrm{~s}^{-1}\right.$, cf. Deforest \& Gurman 1998; Lites et al. 1999). This velocity is representative of the complete sample as there is only $15 \mathrm{~km} \mathrm{~s}^{-1}$ variance
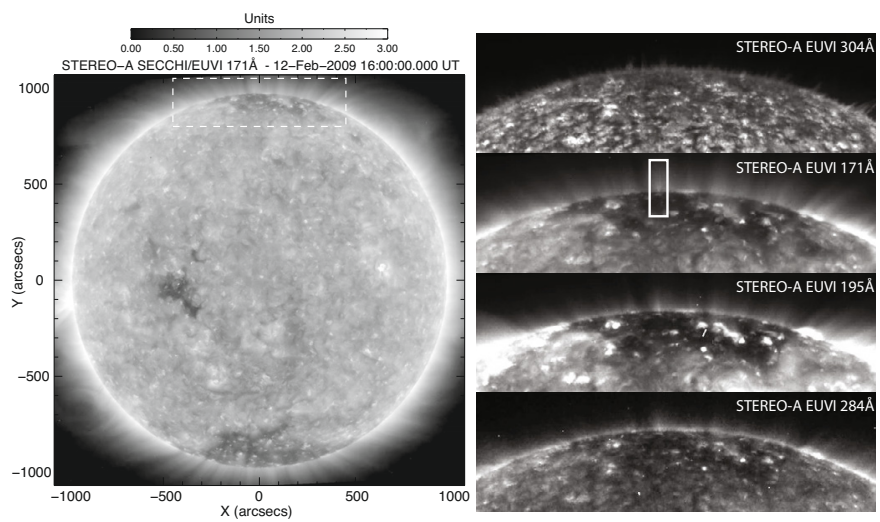

Fig. 1. Context of the observations provided by the STEREO-A SECCHI/EUVI $171 \AA$ passband. The North PCH region that provides the focus of the presented analysis is shown in the box. The inset panels on the right of the figure show the first $304 \AA$, $171 \AA$, $195 \AA$, and $284 \AA$ images of the February 122009 sequence. Also shown in the inset panels are the location of the synthetic slit used to generate the timeseries shown in Fig. 2. The online edition of the journal has movies in support of the four inset panels.

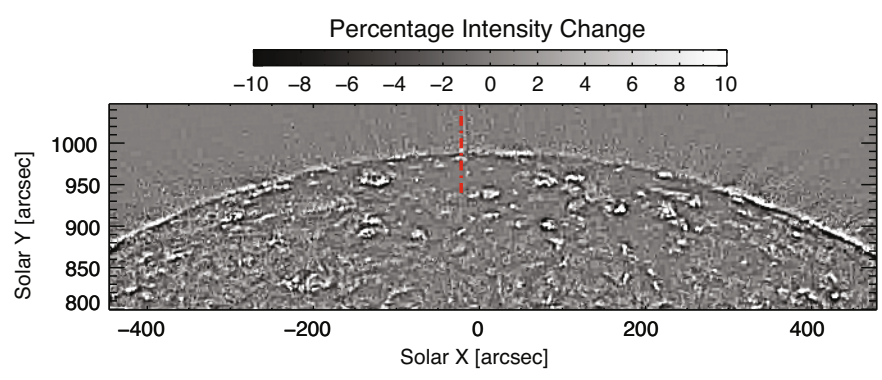

Fig. 2. Example frame from the background-subtracted STEREO-A $171 \AA$ sequence. The synthetic slit used to compute the timeseries shown in Fig. 3 is shown as a red dot-dashed line. The online edition of the journal has a movie in support of this figure.

throughout the four hours of observation, indicating that the apparent motion is a function of the plume's thermodynamic or magnetic properties. The occurrence of these disturbances can be determined by taking a spatially-averaged cut through the slit timeseries $\left(y=990-1000^{\prime \prime}\right.$; dot-dashed region in the top panel) and shown in the center panel. We see that the occurrence of the disturbances is roughly quasi-periodic with events occurring anywhere from 5 to $\sim 15 \mathrm{~min}$ apart. Their strength or amplitude shows less variability ( $\sim 6 \%$ enhancement) as we have noted above. The quasi-periodic nature of the occurrence of these disturbances can be viewed in another way by computing the wavelet power spectrum of the timeseries as is shown in the bottom panel of Fig. 3. The regions of the wavelet power spectrum enclosed in contours are those which have $95 \%$ statistical significance (Torrence \& Compo 1998). The wavelet power spectrum confirms our visual inspection of the spatial cut through the timeseries - it shows a broad range of significant periods, but one of predominantly $15-18 \mathrm{~min}(\sim 1 \mathrm{mHz})$ for a large part of the timeseries. Such quasi-periodicities have been noted before by Deforest \& Gurman (1998) and Banerjee et al. (2000, 2009) where they were interpreted as belonging to propagating slow-mode magneto-acoustic waves.

Using a tool like the xslice routine (publically available in the SolarSoft IDL analysis tree) permits the exploration of the $171 \AA$ image sequences from both spacecraft, in both polar 

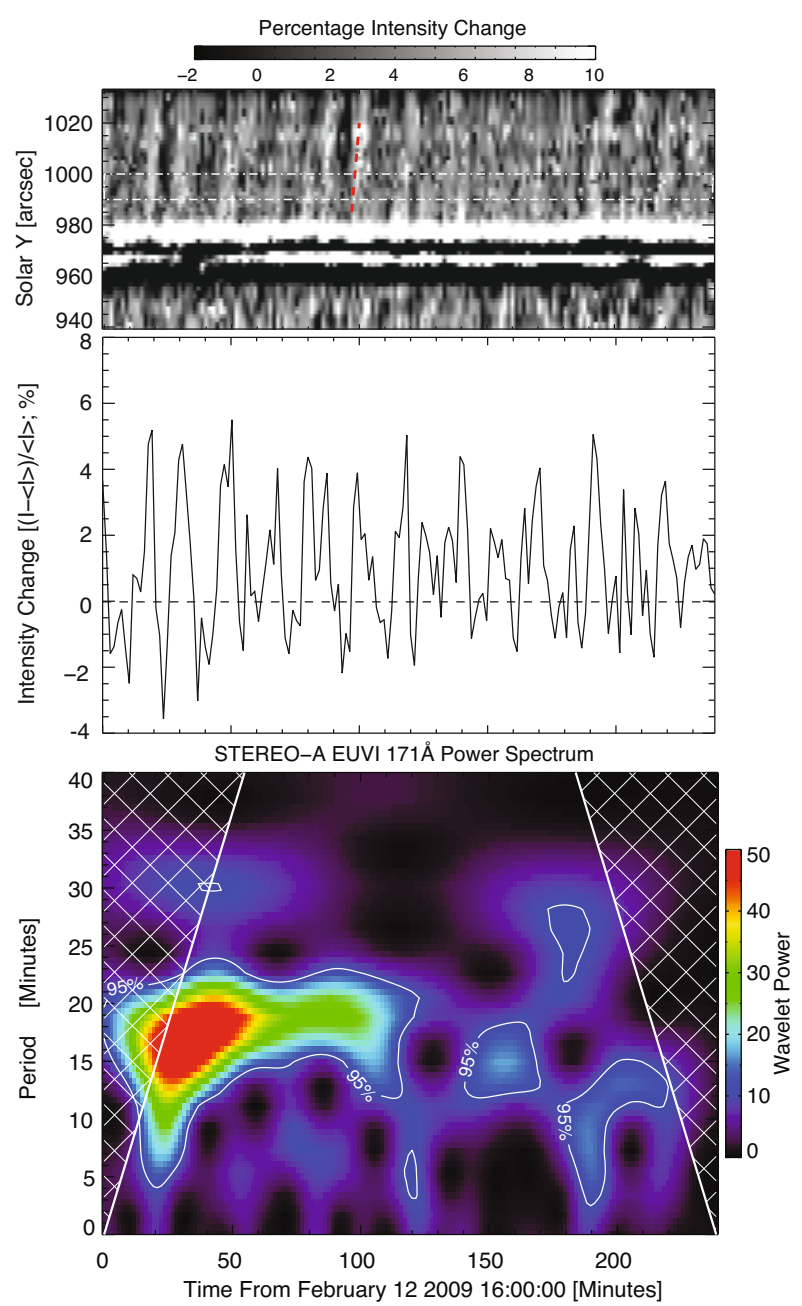

Fig. 3. Timeseries analysis of a plume in the STEREO-A $171 \AA$ image sequence derived from the synthetic slit position shown in Fig. 2. From top to bottom we see the percentage change in the backgroundsubtracted timeseries imaged along the slit, a spatially-averaged cut through the slit timeseries (dot-dashed region in the top panel), and the wavelet power spectrum and its 95\% confidence level (white contours).

regions, and to characterize the appearance of ten clearly defined plumes present in the data. With the method of McIntosh $\&$ Smillie (2004) to determine the plume repeat times we characterize the wavelet power spectra and we see plume disturbance periods in a range of $16.6( \pm 6.6) \mathrm{min}$. For the ten plumes studied in detail we can easily discern 123 individual events, and Fig. 4 shows the derived apparent motions. The distribution of their velocities has a mean of $134( \pm 14) \mathrm{km} \mathrm{s}^{-1}$ where the width of the distribution incorporates the error in computing the gradients in the $\mathrm{x}-\mathrm{t}$ plots.

Finally, very close inspection of the movie supporting Fig. 2 suggests the presence of a ubiquitous, very weak, fine scale, rapidly evolving "mist" of upward propagating companions to the plumes that cover the entirety of the coronal hole limb. Unfortunately, the spatial resolution and compression of the STEREO spacecraft limit any precise diagnosis of this signal and we must wait for the higher signal-to-noise and spatial resolution images of the Solar Dynamics Observatory's (SDO) Atmospheric Imaging Assembly (AIA) for further insight.

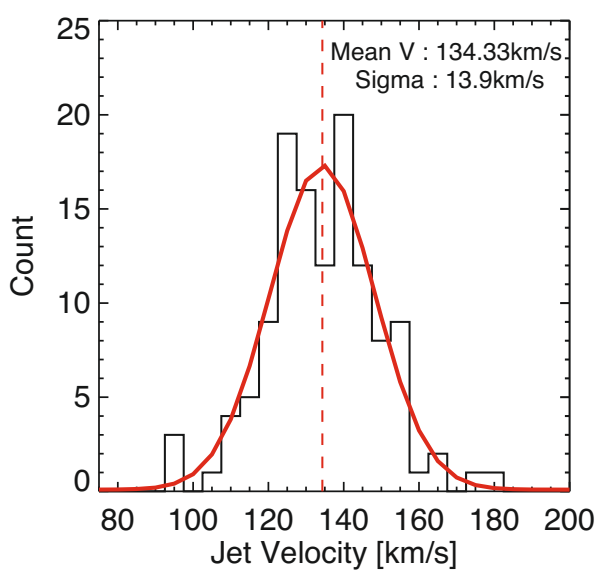

Fig. 4. Distribution of jet velocities clearly visible in the ten plumes characterized. The sample includes information derived from 123 individual jets (see e.g., Fig. 3).

\section{Discussion}

We have observed weak, quasi-periodic, high-speed perturbations in several polar plumes observed by the STEREO spacecraft at a range of temperatures from 0.6-1.5 MK. These perturbations have very similar properties to those observed in active regions (McIntosh \& De Pontieu 2009a), coronal holes (De Pontieu et al. 2009; McIntosh, et al. 2010), and quiet Sun (McIntosh \& De Pontieu 2009b). In each case, these perturbations have been connected spectroscopically to a strong upflowing, weak emission component at the magnetic footpoints. The spectroscopically determined upflows appear to be rooted in dynamic "type-II" spicules in the upper chromosphere (De Pontieu et al. 2009; McIntosh \& De Pontieu 2009a). The overwhelming similarity between these results leads us to propose that the quasi-periodically forced jets observed in polar plumes have a similar cause and, as such, are responsible for loading a significant amount of heated $(\geq 1 \mathrm{MK})$ plasma into the fast solar wind along the open magnetic field lines (Parker 1991), a hypothesis supported by high latitude Ulysses/SWOOPS observations (Yamauchi et al. 2003).

We observe the same types of jets for a wide range of temperatures, from 0.6 to $1.5 \mathrm{MK}$. Because the STEREO data are not taken exactly simultaneously, is not clear whether cool and hot jets co-occur within the same pixel. However, the analysis of McIntosh \& De Pontieu (2009a) of co-located upflows for a wide range of temperatures, as well as observations of largescale jets in coronal holes that appear in simultaneous observations at 140000 and $630000 \mathrm{~K}$ (Scullion et al. 2009) suggest that these events may well be truly multi-thermal. Observations with SDO/AIA will shed light on this issue, one that has important implications for the driving mechanism of these jets, although we expect that they are triggered by the complex interactions of the small scale magnetic flux elements at the base of the plume and its immediate surroundings (Gabriel et al. 2009; Heggland et al. 2009).

It is only appropriate to note that our interpretation in terms of field-directed, high-speed, quasi-periodically triggered upflows is contrary to the widely held interpretation that this observational phenomena is due to compressive (slowmode) magneto-acoustic waves traveling along the plumes (e.g., Deforest \& Gurman 1998; Ofman et al. 1999, 2000; Banerjee et al. 2000; Nakariakov 2006; Banerjee et al. 2009). Unfortunately, the problem is ill-posed, and both interpretations 
are limited by the high inclination angles that polar plumes present relative to our typical line of sight on the Sun-Earth line. Further complications arise from: the commonality of the chromospheric Alfvén and coronal sound speeds $\left(\sim 100 \mathrm{~km} \mathrm{~s}^{-1}\right)$; the weak density enhancements due to the upflow, or wave, passage that are largely lost in the lower part of the plume against the emission from the brighter core of the line, only becoming more prevalent as the background emission drops away exponentially with radial distance (Ofman et al. 1999); and issues persist as to why these objects can have such long "periods" relative to the typical timescales in the lower atmosphere of only a few minutes if the waves come from, or transit through, the lower atmosphere (see e.g., McIntosh et al. 2008; Wang et al. 2009). We suggest that efforts should be made to identify plumes in equatorial coronal holes where they can be studied spectroscopically, down their long axis, in order to check the properties of the line emission with the highest possible signal-to-noise. Such observations are planned using detailed Hinode SOT and EIS observations (using very deep exposures of the coolest lines in its wavelength range) with corresponding STEREO observations at the limb necessary to complete the connection of the plasma from the chromospheric to coronal domain as well as address the wave/upflow interpretation issue. We will investigate the temporal variability in the line core intensity, Doppler shift and broadening down the axis of the plume (studying the phase relationships between them as a wave diagnostic, e.g., Lites \& Chipman 1979), and check for the presence of blue-wing asymmetries (consistent with the presence of a spicule-related upflows De Pontieu et al. 2009). Of course, on disk, we will need to exploit new upper chromospheric diagnostics from SOT to analyze the dynamic behavior (e.g., Rouppe van der Voort et al. 2009).

Should the observed phenomena be related to quasi-periodic mass-loading events it is natural to expect that the change in tension on that magnetic field line will also trigger a quasi-periodic Alfvén wave in the plume (see also Lites et al. 1999). This speculation is supported by the observational evidence of the ubiquitous (and significant) Alfvén wave flux carried by the typeII spicules (De Pontieu et al. 2007b). The presence of these Alfvénic wave motions in the plumes may also go a long way to explaining the enigmatic plume-interplume ${ }^{1}$ relationship of emission line widths (e.g., Hassler et al. 1997; Wilhelm 2000). Finally, we speculate that because the (Alfvén) phase speed of the waves is significantly higher than the field-aligned upflows, the mass on the field line can lead to ideal reflecting conditions for the formation of a turbulent cascade of the Alfvénic wave energy into the plasma that is needed to accelerate the wind (e.g., Velli 1993; Matthaeus et al. 1999; Verdini et al. 2010). It is likely that precise spectroscopic imaging experiments (e.g., Tomczyk et al. 2007) must be made in polar regions to accurately investigate the propagation and magnitude of Alfvén waves in the plume and inter-plume regions.

\section{Conclusion}

We have studied several high cadence STEREO sequences of polar coronal holes at the limb. In all coronal passbands, we observed high speed jets of plasma traveling along the plume structures with a mean velocity of $\sim 135 \mathrm{~km} \mathrm{~s}^{-1}$ at a range of temperatures from 0.5-1.5 MK. These jets have an apparent brightness enhancement of $\sim 5 \%$ above that of the plumes they travel on and repeat quasi-periodically with repeat-times ranging from five to $25 \mathrm{~min}$. These jets are remarkably similar in magnitude, periodicity and velocity to those observed in other magnetic regions of the solar atmosphere. They are multi-thermal in nature. Further, based on the previous results that motivated this study (De Pontieu et al. 2009; McIntosh \& De Pontieu 2009a,b), we speculate that these jets originate in the upper chromosphere (or transition region) and that their quasi-periodic nature can be a ready source of mass and Alfvénic wave energy which can selfconsistently form and accelerate plasma in the fast solar wind.

Acknowledgements. We have benefited and are grateful for frequent discussions with Joe Gurman and Marco Velli. This work was started at a workshop in ISSI, Bern "Small-scale transient phenomena and their contribution to coronal heating". B.D.P., S.W.M., and R.J.L were supported by NASA grants NNX08AH45G, NNX08BA99G, and NNH08CC02C. The EUVI data used here were produced by an international consortium of the Naval Research Laboratory (USA), Lockheed Martin Solar and Astrophysics Lab (USA), NASA Goddard Space Flight Center (USA), Rutherford Appleton Laboratory (UK), University of Birmingham (UK), Max-Planck-Institut for Solar System Research (Germany), Centre Spatiale de Liege (Belgium), Institut d'Optique Theorique et Appliquée (France), and Institut d'Astrophysique Spatiale (France). STEREO is a project of NASA. NCAR is sponsored by the National Science Foundation.

\section{References}

Banerjee, D., O’Shea, E., \& Doyle, J. G. 2000, Sol. Phys., 196, 63 Banerjee, D., Teriaca, L., Gupta, G. R., et al. 2009, A\&A, 499, L29 DeForest, C. E., \& Gurman, J. B. 1998, ApJ, 501, L217

DeForest, C. E., Hoeksema, J. T., Gurman, J. B., et al. 1997, Sol. Phys., 175, 393 De Pontieu, B., McIntosh, S., Hansteen, V. H., et al. 2007a, PASJ, 59, 655 De Pontieu, B., McIntosh, S. W., Carlsson, M., et al. 2007b, Science, 318, 1574 De Pontieu, B., McIntosh, S. W., Hansteen, V. H., et al. 2009, ApJ, 701, L1 Doschek, G. A., Feldman, U., Laming, J. M., et al. 2001, ApJ, 546, 559 Gabriel, A. H., Bely-Dubau, F., \& Lemaire, P. 2003, ApJ, 589, 623 Gabriel, A., Bely-Dubau, F., Tison, E., et al. 2009, ApJ, 700, 551 Hassler, D. M., Wilhelm, K., Lemaire, P., et al. 1997, Sol. Phys., 175, 375 Heggland, L., De Pontieu, B., \& Hansteen, V. H. 2009, ApJ, 702, 1 Lites, B. W., \& Chipman, E. G. 1979, ApJ, 231, 570

Lites, B. W., Card, G., Elmore, D. F., et al. 1999, Sol. Phys., 190, 185 Matthaeus, W. H., Zank, G. P., Oughton, S., et al. 1999, ApJ, 523, L93 Mazzotta, P., Mazzitelli, G., Colafrancesco, S., et al. 1998, A\&AS, 133, 403 McIntosh, S. W., \& Smillie, D. G. 2004, ApJ, 604, 924

McIntosh, S. W., Davey, A. R., Hassler, D. M., et al. 2007, ApJ, 654, 650 McIntosh, S. W., de Pontieu, B., Tomczyk, S., et al. 2008, Sol. Phys., 252, 321 McIntosh, S. W., \& De Pontieu, B. 2009a, ApJ, 706, L80

McIntosh, S. W., \& De Pontieu, B. 2009b, ApJ, 707, 524 McIntosh, S. W., Leamon, R. J., \& De Pontieu, B. 2010, ApJ, submitted Nakariakov, V. M. 2006, Phil. Trans. A, 364, 473

Newkirk, G. J., \& Harvey, J. 1968, Sol. Phys., 3, 321

Ofman, L., Nakariakov, V. M., \& DeForest, C. E., 1999, ApJ, 514, 441

Ofman, L., Romoli, M., \& Poletto, G. 2000, ApJ, 529, 592

Parker, E. N. 1991, ApJ, 372, 719

Rouppe van der Voort, L., Leenaarts, J., de Pontieu, B., et al., 2009, ApJ, 705, 272

Schrijver, C. J., Title, A. M., Berger, T. E., et al. 1999, Sol. Phys., 187, 261 Scullion, E., Popescu, M. D., Banerjee, D., et al. 2009, ApJ, 704, 1385 Torrence, C., \& Compo, G. P. 1998, BAMS, 79, 61

Tomczyk, S., McIntosh, S. W., Keil, S. L., et al. 2007, Science, 317, 1192 Velli, M. 1993, A\&A, 270, 304

Verdini, A., Velli, M., \& Matthaeus, W. H. 2010, ApJ, 708, L116 Wang, H. 1998, ApJ, 509, 461

Wang, T. J., Ofman, L., Davila, J. M., et al. 2009, A\&A, 503, L25 Wilhelm, K. 2000, A\&A, 360, 351

Yamauchi, Y., Suess, S. T., \& Sakurai, T. 2003, Solar Wind Ten, 679, 255

\footnotetext{
1 If the input Alfvén wave flux in plume and inter-plume regions is similar, the higher density of the plume structure (Doschek et al. 2001) naturally would lead to reduced transverse wave amplitudes and thus lower line widths in the plume relative to the surrounding plasma.
} 